\title{
A gut reaction
}

\section{Novel concerns and recommendations to address inappropriate antibiotic use}

\author{
Dalia Hasan, Kristen Reilly \\ Faculty Reviewer: Cathy Faulds, MD, CCFP, FCFP (Department of Family Medicine)
}

\section{ABSTRACT}

The development of antibiotics is one of the greatest advances of modern medicine. While antibiotics have dramatically improved morbidity and mortality rates worldwide, current evidence asserts that one should err on the side of caution when prescribing antibiotics. The medical literature is accumulating studies on alarming consequences of inappropriate antibiotic use. Due to antibiotic overexposure, a North American "hypervirulant" strain of Clostridium difficile has emerged causing more severe gastrointestinal manifestations than previous strains. More recently, antibiotic overuse has been associated with obesity and diabetes mellitus. Unnecessary antibiotic use has led to increasing rates of bacterial resistance rendering more antibiotics ineffective. We are currently on the brink of an era which could reverse all the progress made with the introduction of antibiotics. The salient health consequences of inappropriate antibiotic prescriptions and the threat of a postantibiotic era command attention to practical initiatives that improve antibiotic prescribing patterns.

\section{INTRODUCTION}

The advent of antibiotics revolutionized the way medicine is practiced. Our most effective tools for inhibiting bacterial growth or killing bacteria are these powerful pills. When first introduced in 1928 , antibiotics could effectively treat any bacteria they were targeting. These wonder drugs were particularly brilliant alternatives to invasive surgeries. Morbidity and mortality rates have dramatically decreased since the development of antibiotics, increasing life expectancy by 15 years, and saving millions of lives. ${ }^{1}$

Unfortunately, such a radical change in medicine has brought with it many adverse effects. Inappropriate antibiotic prescription is a serious concern as it opens the door to numerous health sequelae. Antibiotics have been known to cause Clostridium difficile infections by disrupting the beneficial microbes that protect our gut. ${ }^{2}$ Through a similar mechanism, new research has now linked the inappropriate use of antibiotics with increased risk of chronic diseases, such as diabetes mellitus and obesity. ${ }^{1,3-5}$ The era of untreatable infections is impending if bacteria continue to become increasingly resistant to first-line antibiotic therapy. ${ }^{1,6}$ The Chief Medical Officer for England has described this potential postantibiotic era as "apocalyptical" and "catastrophic."

In light of these findings, this article will bring forth new evidence on the adverse effects of antimicrobials, and advocate for their judicious use with practical recommendations.

\section{ANTIBIOTIC OVERUSE}

The evidence of overexposure to antibiotics in Canada is overwhelming. Studies have shown that in Canadian hospitals, only half of antibiotic prescriptions were justifiably used. ${ }^{7,8}$ Consequently, a staggering loss of up to $\$ 850$ million, yearly, is attributed to inappropriate antibiotic use in Canada. ${ }^{7}$ In order to address this problem, it is important to recognize why antibiotics are excessively prescribed. In the context of diagnosing lower respiratory tract infections, a study conducted with the College of Family Physicians of Canada examined some possible causes of the misuse of antibiotics. ${ }^{9}$ This study determined that physicians were (1) uncertain about the need for antibiotics, (2) conflicted about the clinical diagnosis, (3) concerned that patients could become sicker, and (4) pressured by patients or parents to prescribe antibiotics. This is especially disconcerting as a public opinion poll demonstrated that $50 \%$ of Canadians are misinformed about the effectiveness of antibiotics on viruses. ${ }^{7}$ The etiology for antibiotic prescribing must be addressed to circumvent alarming health concerns.

\section{HEALTH CONSEQUENCES}

Understanding the significance of taking unnecessary antibiotics can help prevent their overuse. An emerging field of microbiology research focuses on the relationship between antibiotics and gut microbiota, the community of bacteria that reside within the gastrointestinal tract (formerly known as gut flora). Gut microbiota are able to counteract infection by playing an essential role in immune system development. ${ }^{10}$ Various antibiotics can alter the beneficial microbiota in the colon, leaving it vulnerable to infection. ${ }^{11}$ High doses of or prolonged exposure to antibiotics can lead to an increased risk of $C$. difficile infections, which range from causing severe diarrhea to life-threatening pseudomembranous colitis. ${ }^{2}$ Of note, a major article has recently reported a North American "hypervirulant" strain which causes increasingly severe $C$. difficile infections. ${ }^{12}$ This is particularly disturbing as C. difficile is currently a leading cause of nosocomial infection, ${ }^{11}$ highlighting the need to correctly monitor antibiotic dosage and duration to maintain the integrity of the microbiota.

A lesser known function of the microbiota is its role in metabolism. Current research has identified additional features to its role in immune defense, including the regulation of fat storage, hormone secretion, and energy expenditure. ${ }^{1,10}$ While it is known that antibiotics can suppress or eradicate gut microbiota, their influence on the microbiota's nutrient metabolism has been associated with an increased risk of type 1 and type 2 diabetes mellitus. ${ }^{1,3}$ The disturbance in glucose homeostasis and insulin sensitivity has been suspected to be caused by use of oral vancomycin, bactericidal, and 
narrow-spectrum antibiotics. ${ }^{10,13}$ It is important to note that these are preliminary studies in this field, and further evidence would be needed to make conclusive assertions.

Another chronic disease that has been linked to prolonged antibiotic use is obesity. In mice, exposure to antibiotics has been shown to increase body fat mass due to disruption of the microbiota composition. ${ }^{14}$ Antibiotics have also been shown to contribute to the risk of obesity in humans; some studies go on to indicate that people who are obese have lower microbial diversity. ${ }^{4,5,15}$ The postnatal period is especially susceptible to disruptions of the microbial environment from antibiotics, as the gut microbiota in infants is still developing. ${ }^{16}$ Azad and colleagues observed that boys who took antibiotics before their first birthday were 5 times more likely to become overweight by age $12 .{ }^{16}$ The mechanism is believed to be similar to that seen in agriculture where livestock given antibiotics at a young age improve feeding and weight gain due to growth promotion. $^{17}$

The heavy introduction of antibiotics in agricultural practice was one of the first settings to raise a concern for antibiotic resistance. ${ }^{15}$ It has been well known for decades that unnecessary antibiotic use can lead to resistance, but the types of bacteria that are evolving to evade the life-saving effects of antimicrobials are increasingly becoming more prevalent. Methicillin-resistant Staphylococcus aureus (MRSA) is a Gram-positive bacterium that is the archetype of antimicrobial resistance but there are now new "bugs on the block" that disarm Gram-negative antibiotics. The emergence of Gram-negative antibiotic resistance leads to dire consequences as without effective antimicrobials, they are capable of causing high rates of morbidity and mortality. ${ }^{18}$ New Delhi Metallo-beta-lactamase-1 (NDM-1) is a newly discovered enzyme found in Gram-negative bacteria that is able to defy antibiotics which are normally used to target Gram-negative organisms. NDM-1 can be found in Escherichia coli bacteria which are becoming more difficult to treat due to the unavailability of alternative antimicrobials. ${ }^{18}$ Antibiotic resistance has now become a public health emergency as it is predicted that the role of antibiotics in the eradication of disease will become obsolete in as little as 20 years. ${ }^{6,16}$

\section{PRACTICAL SOLUTIONS}

Given the infancy of research on the effect of antibiotics on gut microbiota, mitigating the adverse effects of the specific emerging health consequences discussed above is still a challenge.

The literature indicates that fecal microbiota transplants (FMT), well known for their effective treatment of C. difficile, are now being piloted as a therapeutic strategy for obesity. ${ }^{15}$ Early studies show that this novel approach, involving FMT from lean to obese patients, has been effective in improving insulin resistance..$^{15}$ Furthermore, probiotics may be a useful treatment for metabolic diseases like obesity and diabetes as they have been shown to reduce adiposity, body fat, and weight gain. ${ }^{19}$ Probiotics may be administered in multiple forms (ie, capsule, sachet, or yogurt), and are currently available in supermarkets for consumption without a prescription. ${ }^{20,21}$ Finally, there is a critical need for new and modified forms of antibiotics to treat the diversifying and increasing number of resistant bacteria, particularly targeted at Gram-negative bacteria. ${ }^{1}$

More practical recommendations for physicians to address the inappropriate prescription of antibiotics are numerous; however, 3 specific examples will be discussed. The first, antimicrobial stewardship programs (ASP), are "coordinated interventions designed to improve and measure the appropriate use of antimicrobial agents by promoting the selection of the optimal antimicrobial drug regimen including dosing, duration of therapy, and route of administration." 22 The implementation of patient and/or provider ASP education programs has led to substantial reductions (up to $35 \%$ ) of inappropriate antibiotic prescriptions..$^{23,24}$ Recent evidence suggests, however, that even simpler and less costly ASP interventions can result in a significant decline in antibiotic prescription. ${ }^{25}$ Meeker and colleagues found that physicians who displayed a signed letter of commitment to avoid inappropriate prescriptions of antibiotics in examinations rooms for 12 weeks reduced their antibiotic prescriptions by nearly $20 \%$. While physicians' commitment to ASP is pivotal to their success, their uncertainty in the infectious etiology (ie, bacterial or viral) is a major contributing factor to the inappropriate prescription of antibiotics. ${ }^{9}$ To address this challenge, it is recommended that where appropriate, physicians are vigilant about ordering cultures and perform throat swabs and rapid testing to confirm diagnoses. ${ }^{9}$ Lastly, physicians should exercise more preventive medicine such as prophylaxis for influenza given that patients who present with influenza-like symptoms are often inappropriately prescribed with antibiotics. ${ }^{26,27}$ A study showed that $38 \%$ of health care visits for influenza resulted in an antibiotic prescription. ${ }^{27}$ Therefore, more efforts in promoting flu vaccinations would decrease the spread of influenza infections altogether, and subsequently reduce the proportion of unnecessary antibiotic use. ${ }^{26}$

\section{CONCLUSION}

Cautious and thoughtful use of antibiotics remains paramount to the prevention of antibiotic-acquired illnesses. However, it is now becoming more evident that antibiotics can assault the human microbiome causing a myriad of adverse and potentially chronic health effects. Additional research is necessary to establish alternative evidence-based strategies that foster the maintenance of normal and healthy gut microbiota. More immediately, there is strong evidence supporting tangible initiatives that improve antibiotic prescribing patterns, which can immediately be adopted by physicians for the best practice of care. 


\section{REFERENCES}

1. Keeney KM, Yurist-Doutsch S, Arrieta M-C, Finlay BB. Effects of antibiotics on human microbiota and subsequent disease. Annu Rev Microbiol. 2014;68(1):217-35.

2. Stevens V, Dumyati G, Fine LS, Fisher SG, Van Wijngaarden E. Cumulative antibiotic exposures over time and the risk of clostridium difficile infection. Clin Infect Dis. 2011;53(1):42-8.

3. Mikkelsen KH, Knop FK, Frost M, Hallas J, Pottegård A. Use of antibiotics and risk of type 2 diabetes: a population-based case-control study. J Clin Endocrinol Metab. 2015 Oct;100(10):3633-40.

4. Jernberg C, Löfmark S, Edlund C, Jansson JK. Long-term impacts of antibiotic exposure on the human intestinal microbiota. Microbiology. 2010;156(Pt 11):3216-23

5. Bailey LC, Forrest CB, Zhang P, Richards TM, Livshits A, DeRusso PA. Association of antibiotics in infancy with early childhood obesity. JAMA Pediatr. 2014;168(11):1063-9.

6. Viens AM, Littmann J. Is antimicrobial resistance a slowly emerging disaster? Public Health Ethics. 2015 Nov; 8(3): 255-65.

7. Thomas RE. Controlling antimicrobial resistance Health Canada proposes an integrated action plan for Canadians. 1998;44:1901-2.

8. Schollenberg E, Albritton WL. Antibiotic misuse in a pediatric teaching hospital. Can Med Assoc J. 1980;122(1):49-52.

9. McIsaac WJ, To T. Antibiotics for lower respiratory tract infections. Still too frequently prescribed? Can Fam Physician. 2004;50:569-75.

10. Sommer F, Bäckhed F. The gut microbiota - masters of host development and physiology. Nat Rev Microbiol. Nature Publishing Group; 2013;11(4):227-38.

11. Seekatz AM, Young VB. Clostridium difficile and the microbiota. J Clin Invest. 2014;124(10):4182-9.

12. See I, Mu Y, Cohen J, Beldavs ZG, Winston LG, Dumyati G, et al. NAP1 strain type predicts outcomes from Clostridium difficile infection. Clin Infect Dis. 2014;58(10):1394-400.

13. Vrieze A, Out C, Fuentes S, Jonker L, Reuling I, Kootte RS, et al. Impact of oral vancomycin on gut microbiota, bile acid metabolism, and insulin sensitivity. J Hepatol. European Association for the Study of the Liver; 2014;60(4):824-31.

14. Cho I, Yamanishi S, Cox L, Methé BA, Zavadil J, Li K, et al. Antibiotics in early life alter the murine colonic microbiome and adiposity. Nature. 2012;488(7413):621-6.

15. Konturek PC, Haziri D, Brzozowski T, Hess T, Heyman S. Emerging role of fecal microbiota therapy in the treatment of obesity. J Physiol Pharmacol. 2015 Jul;66(4):483-91.

16. Azad MB, Bridgman SL, Becker AB, Kozyrskyj AL. Infant antibiotic exposure and the development of childhood overweight and central adiposity. Int J Obes (Lond). Nature Publishing Group; 2014;38(10):135.

17. Jukes TH. Antibiotics in animal feeds and animal production. Bioscience. 1972;22(9):526-34.

18. Kaye KS, Pogue JM. Infections caused by resistant Gram-negative bacteria : epidemiology and management. Pharmacotherapy. 2015 Oct;35(10):949-62.

19. Mekkes M, Weenen T, Brummer R, Claassen E. The development of probiotic treatment in obesity: a review. Benef Microbes. 2014;5(1):1928.

20. D'Souza AL, Rajkumar C, Cooke J, Bulpitt CJ. Probiotics in prevention of antibiotic associated diarrhoea: meta-analysis. BMJ. 2002:324(7350):1361.

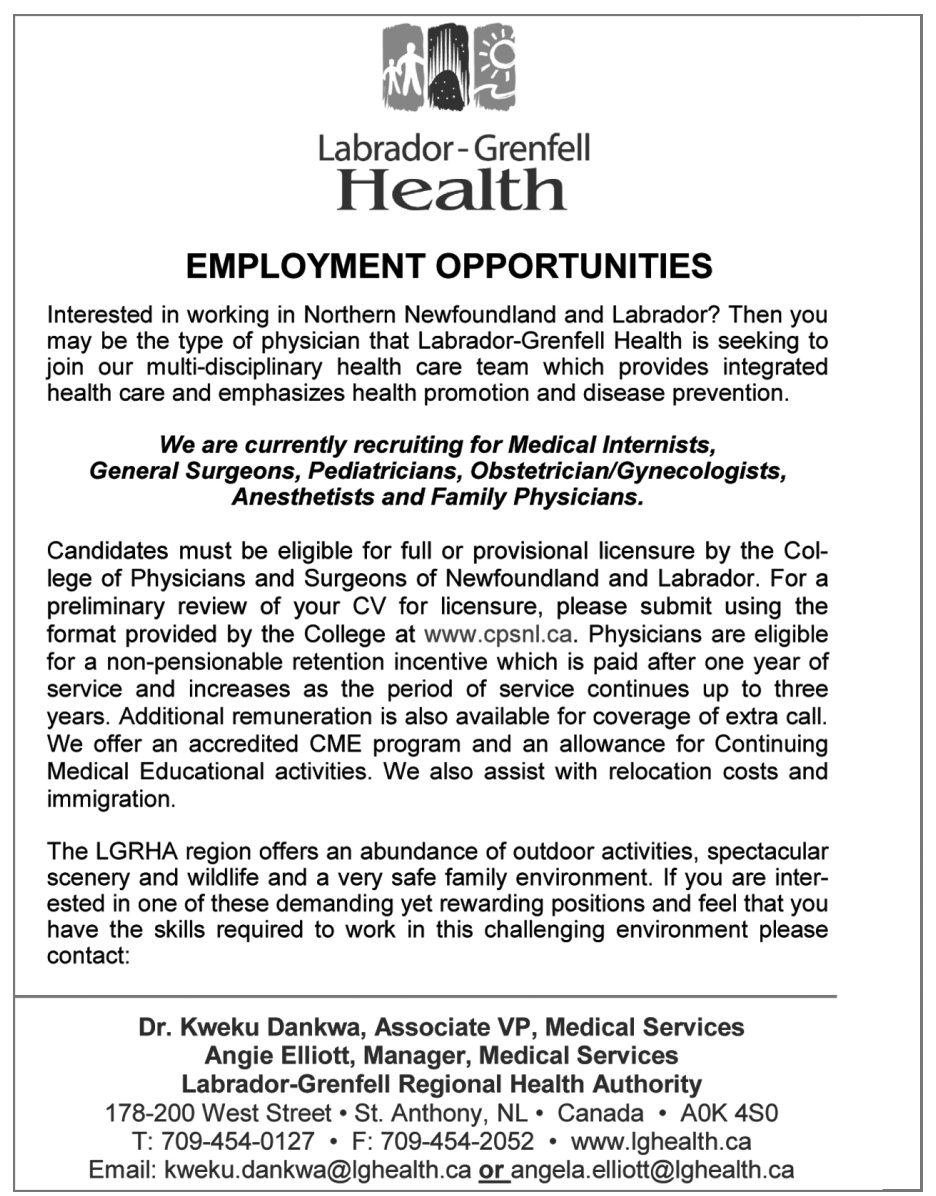

21. Johnston BC, Supina AL, Ospina M, Vohra S. Probiotics for the prevention of pediatric antibiotic-associated diarrhea gastrointestinal and extra-gastrointestinal disease. (Review). Cochrane Database Syst Rev. 2011 Nov 9;(11):CD004827

22. Fishman N. Policy statement on antimicrobial stewardship by the Society for Healthcare Epidemiology of America (SHEA), the Infectious Diseases Society of America (IDSA), and the Pediatric Infectious Diseases Society (PIDS). Infect Control Hosp Epidemiol. 2012;33(04):322-

23. Canadian Antimicrobial Resistance Alliance. Comprehensive overview of antibiotic resistance in Canada. 2006 [cited 2015 Nov 15]. p. 1-27.

24. Roque F, Herdeiro MT, Soares S, Teixeira Rodrigues A, Breitenfeld L, Figueiras A. Educational interventions to improve prescription and dispensing of antibiotics: a systematic review. BMC Public Health. 2014;14(1):1276.

25. Meeker D, Knight TK, Friedberg MW, Linder JA, Goldstein NJ, Fox $\mathrm{CR}$, et al. Nudging guideline-concordant antibiotic prescribing. JAMA Intern Med. 2014;174(3): 425-31.

26. Low D. Reducing antibiotic use in influenza: challenges and rewards. Clin Microbiol Infect. European Society of Clinical Infectious Diseases; 2008;14(4):298-306.

27. Ciesla G, Leader S, Stoddard J. Antibiotic prescribing rates in the US ambulatory care setting for patients diagnosed with influenza, 1997-2001. Respir Med. 2004;98(11):1093-101 